\title{
Thermal Bending of the Rotor Due to Rotor-to-Stator Rub
}

\author{
PAUL GOLDMAN*, AGNES MUSZYNSKA and DONALD E. BENTLY
}

Bently Rotor Dynamics Research Corporation, 1711 Orbit Way, Bldg. 1, Minden, NV 89423, USA

(Received 5 September 1997; In final form 21 July 1998)

\begin{abstract}
The rotor thermal bending due to the rotor-to-stator rubbing can lead to one of three types of observed rotor lateral motion: (1) spiral with increasing amplitude, (2) oscillating between rub/no-rub conditions, and (3) asymptotical approach to the rotor limit cycle. Based on the machinery observations, it is assumed in the analytical part of the paper that the speed scale of transient thermal effects is considerably lower than that of rotor vibrations, and that the thermal effect reflects only on the rotor steady-state vibrational response. This response would change due to thermally induced bow of the rotor, which can be considered to slowly vary in time for the purpose of rotor vibration calculations. Thus uncoupled from the thermal problem, the rotor vibration is analyzed. The major consideration is given to the rotor which experiences intermittent contact with the stator, due to predetermined thermal bow, unbalance force, and radial constant load force. In the case of inelastic impact, it causes an on/off, step-change in the stiffness of the system. Using a specially developed variable transformation for the system with discontinuities, and averaging technique the resonance regimes of motion are obtained. These regimes are used to calculate the heat generated during contact stage, as a function of thermal bow modal parameters, which is used as a boundary condition for the rotor heat transfer problem. The latter is treated as quasi-static, which reduces the problem to an ordinary differential equation for the thermal bow vector. It is investigated from the stability standpoint.
\end{abstract}

Keywords: Rotor, Vibrations, Thermal bow, Rub, Averaging technique

\section{INTRODUCTION}

Rotor-to-stator rub, an unwelcome contact between rotating and nonrotating elements of a machine, can be one of the most damaging malfunctions of rotating machinery. Generated by some perturbation of normal operating conditions that causes an increase of rotor vibration level, and/ or an increase of the rotor centerline eccentricity, the rub can maintain itself, and gradually become more severe. The self-generating feature of this phenomenon originates from the interaction between rub-related thermal effects and lateral vibrational response of the rotor. Starting from

\footnotetext{
*Corresponding author. Tel.: (702)782 3611. Fax: (702)7829236. E-mail: psg@,bnc117psg.bently.com.
} 
pioneering works of Taylor (1924) and Newkirk (1926), the unwinding spiral vibrations of rotors are documented in several papers (Black, 1968; Kroon and Williams, 1939; Dimaragonas, 1973; Kellenberger, 1979; Natho and Crenwelge, 1983; Hashemi, 1984; Smalley, 1987; Muszynska, 1993). In addition to the spiral response, Dimaragonas, (1974) described an oscillating mode of shaft vibration, occurring during the transition from the spiraling to a steady-state mode. A similar result from an improved rotor dynamic model was obtained by Muszynska (1993).

The problem of rub-related heat distribution was discussed by several authors, for example, Sweets (1966), Kellenberger (1979) and Smalley (1987). The most complete analysis of the heat transfer problem associated with rub is given in the book by Dimaragonas and Paipetis (1983). In all referenced literature the analysis of the shaft bow, resulting from the uneven temperature distribution due to rub, is based on an approximation on the mean flexural rotation of one end of the shaft in relation to the other (Goodier, 1958).
The idea of the discontinuous variable transformation applied in this paper for the rub dynamics analytical description appeared first in the paper by Zhuravlev (1978), and was expanded later by Petchenev and Fiddling (1992) and Goldman and Muszynska (1994a,b; 1995a,b).

\section{MATHEMATICAL MODEL OF THE RUBBING ROTOR}

An isotropic rotor in its lateral mode motion is considered (Fig. 1). The rub (or more generally any local nonlinearity which creates a thermal effect) occurs at the shaft axial location $l_{2}$. If the rotary inertia and shear stresses are neglected, the equations of motion of the rotor can be expressed as follows:

$$
\begin{aligned}
m \ddot{\vec{r}} & +\frac{\partial^{2}}{\partial l^{2}}\left[E J \frac{\partial^{2}\left(\vec{r}-\vec{\rho}_{T}\right)}{\partial l^{2}}\right] \\
& =m \vec{e} \Omega^{2} \mathrm{e}^{\mathrm{j} \varphi}+\vec{Q}, \quad \mathrm{j}=\sqrt{-1}
\end{aligned}
$$

where $x(l, t), y(l, t)$ are horizontal and vertical displacements of the rotor at the axial location $l$

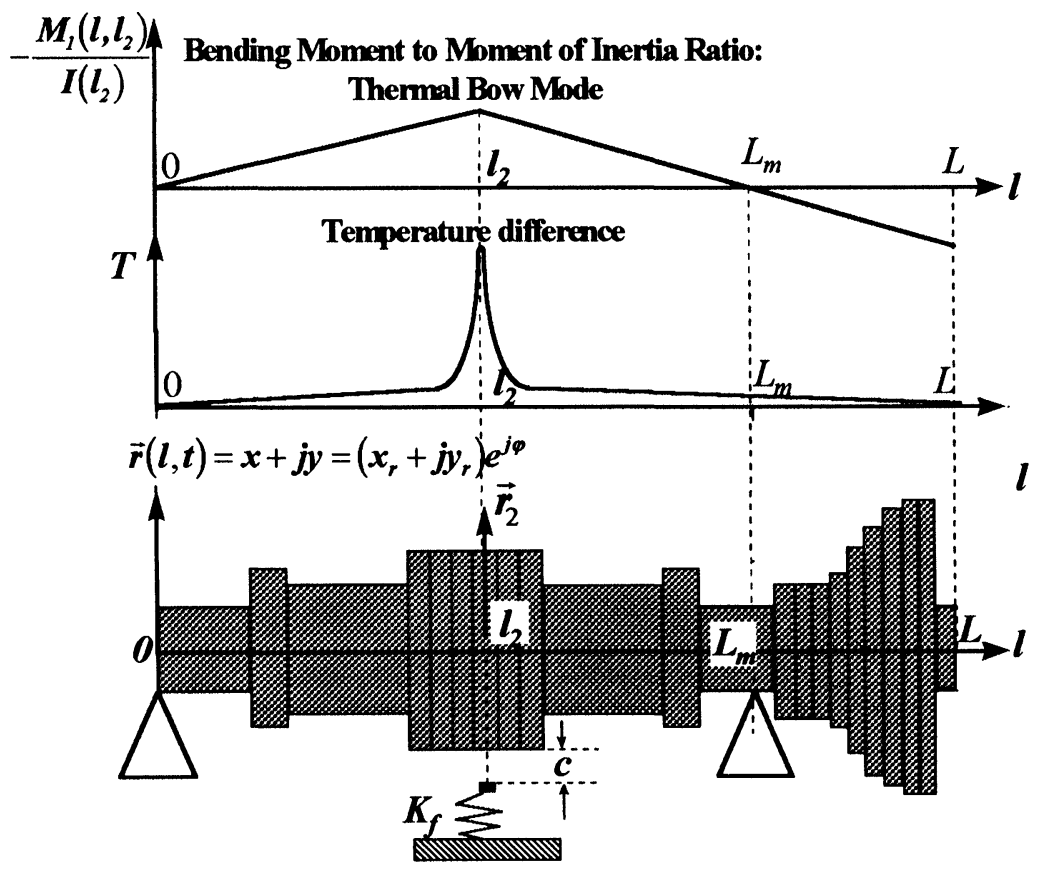

FIGURE 1 Physical model of the rubbing rotor. 
in the stationary coordinates, $\vec{r}=x+\mathrm{j} y ; E J$ is bending stiffness, $m$ is mass linear density, $\vec{\rho}_{T}$ is a vector of thermal bending in stationary coordinates, $\vec{\rho}_{T}(l, t)=\overrightarrow{\hat{\rho}}_{T}(l, t) \mathrm{e}^{\mathrm{j} \varphi}, \overrightarrow{\hat{\rho}}_{T}$ is a vector of thermal bending in rotating coordinates, $\Omega$ is rotative speed, $\varphi=\Omega t+\alpha, \vec{e}$ is a distributed unbalance vector in the coordinate system rotating with the rotor at the particular axial location $l, \vec{Q}=Q_{x}+\mathrm{j} Q_{y}$ is a complex vector describing linear density of the external and nonconservative forces. Assuming that the contact/no-contact situation at the axial location $l_{2}$ generates a radial reaction force

$$
\begin{aligned}
& \vec{N}=-\vartheta K_{f}\left(\left|\vec{r}_{2}\right|-c\right) \frac{\vec{r}_{2}}{\left|\vec{r}_{2}\right|}, \\
& \vartheta=\left\{\begin{array}{ll}
1 & \text { if }\left|\vec{r}_{2}\right| \geq c \\
0 & \text { if }\left|\vec{r}_{2}\right|<c
\end{array},\left|\vec{r}_{2}\right|=\sqrt{x_{2}^{2}+y_{2}^{2}}\right.
\end{aligned}
$$

where $K_{f}$ is the local stiffness of the stationary obstacle, $\vec{r}_{2}=\vec{r}\left(l_{2}, t\right), c$ is the radial clearance between the rotor and the obstacle, the vector $Q$ can be presented in the following form:

$$
\vec{Q}=-C(l) \dot{\vec{r}}+q(l) \mathrm{e}^{\mathrm{j} \gamma(l)}+\frac{1}{L} \delta\left(\frac{l}{L}-\frac{l_{2}}{L}\right) \vec{N}(1+\mathrm{j} f) .
$$

Here $C(l)$ is a damping linear density, $q(l), \gamma(l)$ are radial side-load forces linear density and their angular orientation, respectively, $f$ is a dry friction coefficient, $L$ is the length of the rotor and $\delta(\ldots)$ is the Dirac function. The thermal bow appears due to an uneven temperature distribution along the rotor caused by the friction force-generated heat. The latter can be characterized by the heat rate density $g(l, \psi, t)$ per unit area of the rotor cross section (is an angular coordinate, Fig. 2). Considering the particular area element $R_{2} \mathrm{~d} l \mathrm{~d} \psi\left(R_{2}\right.$ is the rotor external radius), on the rotor surface around the point with axial coordinate $l$, the friction force $\mathrm{j} f \vec{N}$ is applied to the rotor if $\psi_{N}-\mathrm{d} \psi_{N} \leq \psi \leq \psi_{N}+\mathrm{d} \psi_{N}$ and $l_{2}-\mathrm{d} l \leq l \leq l_{2}+\mathrm{d} l$ (it is assumed that the rotor-tostator contact occurs at a single location only), where $\psi_{N}=2 \pi-\varphi+\arg \left(\vec{r}_{2}\right)$ is the angular position of the friction force (Fig. 2). The friction force, therefore, can be expressed in a form of a distribution over the rotor surface as follows:

$$
\begin{aligned}
\vartheta f|\vec{N}|= & \vartheta f \int_{-L / 2}^{L / 2} \int_{0}^{2 \pi} \frac{|\vec{N}|}{L} \delta \\
& \times\left(\frac{l}{L}-\frac{l_{2}}{L}\right) \delta\left(\psi-\psi_{N}\right) \mathrm{d} l \mathrm{~d} \psi .
\end{aligned}
$$

$\delta(\ldots)$ is defined for angles $\psi$ as a periodic with period of $2 \pi$. Since the rotor velocity at the contact location can be approximated as $R_{2} \Omega$, the heat rate density $g(l, \psi, t)$ per unit area equals to the friction force power per unit area. Taking into account Eq. (4), it can be presented as follows:

$$
g=f \vartheta \Omega \frac{|\vec{r}|-c}{L} \delta\left(\frac{l}{L}-\frac{l_{2}}{L}\right) \delta(\psi+\varphi-\arg (\vec{r})) .
$$

The thermal conductivity equation with initial and boundary conditions

$$
\begin{aligned}
& \frac{\partial^{2} T}{\partial r^{2}}+\frac{1}{r} \frac{\partial T}{\partial r}+\frac{1}{r^{2}} \frac{\partial^{2} T}{\partial \psi^{2}}+\frac{\partial^{2} T}{\partial l^{2}}=\frac{1}{\hat{K}} \frac{\partial T}{\partial t}, \\
& \left.T\right|_{t=0}=0, \quad \hat{k} \frac{\partial T}{\partial l}+\left.\hat{h} T\right|_{l=0, L}=0, \\
& \hat{k} \frac{\partial T}{\partial r}+\left.\hat{h} T\right|_{r=R_{2}}=g(l, \psi, t), \quad-\hat{k} \frac{\partial T}{\partial r}+\left.\hat{h} T\right|_{r=R_{1}}=0,
\end{aligned}
$$

describes heating of the shaft due to rub. Here $T=T(r, \psi, l, t)$ is a difference of temperatures

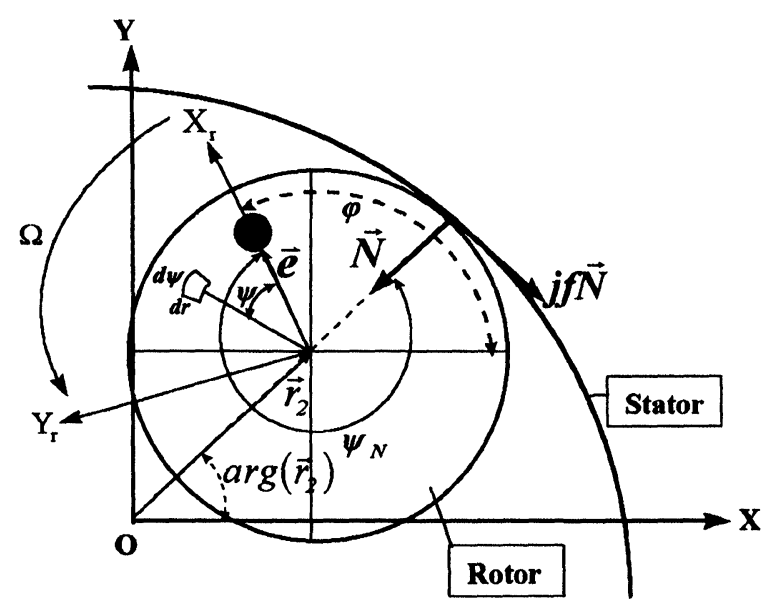

FIGURE 2 Cross-section of the rotor at the rub axial location $l=l_{2}$ 
between the environment and the material point of the shaft with coordinates $l, r, \psi$ at an instant $t$, and $\hat{K}, \hat{k}, \hat{h}$ are thermodynamic constants, $R_{1}$ is the rotor internal radius. To complete the problem formulation, a relationship between the temperature distribution and thermal bow has to be derived. In order to accomplish this, the assumption is made that the rotor can be considered within the limits of Euler's beam theory. In this case, the thermal stress-related bending moments in the rotating coordinate axes $X_{r}, Y_{r}$ are as follows:

$$
\begin{aligned}
& M_{Y}=-\chi_{\mathrm{T}} \int_{0}^{2 \pi} \mathrm{d} \psi \int_{R_{1}}^{R_{2}} E \operatorname{Tr}^{2} \cos \psi \mathrm{d} r=M_{Y}(t, l), \\
& M_{X}=\chi_{\mathrm{T}} \int_{0}^{2 \pi} \mathrm{d} \psi \int_{R_{1}}^{R_{2}} E \operatorname{Tr}^{2} \sin \psi \mathrm{d} r=M_{X}(t, l),
\end{aligned}
$$

where $\chi_{\mathrm{T}}$ is the thermal expansion coefficient, $E$ is Young's modulus of elasticity.

According to the Castigliano's theorem and Eqs. (7), the vector $\vec{\rho}_{T}(l, t)$ of the thermal bow in the rotating coordinates can be expressed in the form of convolution:

$$
\begin{aligned}
& \overrightarrow{\hat{\rho}}_{T}(l, t)=-\chi_{\mathrm{T}} \int_{0}^{L} \frac{M_{1}\left(l, l_{a}\right)}{I\left(l_{a}\right)} \\
& \times\left[\int_{0}^{2 \pi} \mathrm{d} \psi \int_{R_{1}\left(l_{a}\right)}^{R_{2}\left(l_{a}\right)} T\left(t, r, \psi, l_{a}\right) \mathrm{e}^{\mathrm{j} \psi} r^{2} \mathrm{~d} r\right] \mathrm{d} l_{a}, \\
& \overrightarrow{\hat{\rho}}_{T}(l, t) \approx-\chi_{\mathrm{T}} \frac{M_{1}\left(l, l_{2}\right)}{I\left(l_{2}\right)} \overrightarrow{\tilde{T}} \\
& \overrightarrow{\tilde{T}}=\int_{0}^{L} \mathrm{~d} l_{a} \int_{0}^{2 \pi} \mathrm{d} \psi \int_{R_{1}\left(l_{a}\right)}^{R_{2}\left(l_{a}\right)} T\left(t, r, \psi, l_{a}\right) \mathrm{e}^{\mathrm{j} \psi} r^{2} \mathrm{~d} r
\end{aligned}
$$

where $M_{1}\left(l, l_{a}\right)$ is a bending moment at the axial location $l_{a}$, resulting from the unit load applied at the axial location $l$. For simplicity it is assumed that the rotor has constant modulus of elasticity $E$ for all cross-sections along its length. Due to the local character of the rotor heating (Fig. 1) the convolution can be approximated as shown in the second of Eqs. (8). An example of a simply supported rotor with overhung impeller, shown in Fig. 1, has the following expression for $M_{1}\left(l, l_{2}\right)$ :

$M_{1}\left(l, l_{2}\right)=\frac{1}{2}\left[\left|l-l_{2}\right|-\frac{l}{L_{m}}\left|L_{m}-l_{2}\right|-\frac{l_{2}}{L_{m}}\left(L_{m}-l\right)\right]$.

Obviously the expression - $\left(M_{1}\left(l, l_{2}\right) / I\left(l_{2}\right)\right)$ determines the mode shape of the thermal bow (see Fig. 1) while $\overrightarrow{\tilde{T}}(t)$ plays the role of the modal time vector-function. The equations which describe the latter can be derived from Eqs. (6) by integral transformations (see Taylor (1924) for details), with the assumption that all thermal processes are limited to the rotor area with the same cross-section defined by internal radius $R_{1}\left(l_{2}\right)$ and external radius $R_{2}\left(l_{2}\right)$, and averaged over the yet unknown period of mechanical oscillations $(\varepsilon$ is a small parameter):

$$
\frac{\mathrm{d} \overrightarrow{\tilde{T}}}{\mathrm{~d} \tau}=\varepsilon\left(\frac{R_{2}^{3}\langle\overrightarrow{\hat{g}}\rangle}{k_{1}}-\frac{\overrightarrow{\tilde{T}}}{D_{T}}\right)
$$

where

$$
\begin{aligned}
& \langle\overrightarrow{\hat{g}}\rangle=\left\langle\int_{0}^{L} \mathrm{~d} l \int_{0}^{2 \pi} g \mathrm{e}^{\mathrm{j} \psi} \mathrm{d} \psi\right\rangle \\
& =f \Omega K_{f}\left\langle\vartheta\left(\left|\vec{r}_{2}\right|-c\right) \mathrm{e}^{-\mathrm{j}\left(\varphi-\arg \vec{r}_{2}\right)}\right\rangle, \\
& \kappa=\frac{R_{1}\left(l_{2}\right)}{R_{2}\left(l_{2}\right)}, \quad k_{1}=\frac{\hat{k}}{R_{2}}, \\
& D_{T} \\
& =\frac{\left(1-\kappa^{2}\right)\left[k_{1}\left(1+3 \kappa^{2}\right)+\kappa \hat{h}\left(1-\kappa^{2}\right)\right]}{4\left\{\left(1-\kappa^{2}\right)\left(k_{1}+\kappa h\right)+h\left[1+\kappa^{2}+8 \kappa^{3}+\kappa\left(1-\kappa^{2}\right) \frac{h}{k_{1}}\right]\right\}} \\
& \varepsilon=\frac{\hat{K}}{\Omega R_{2}\left(l_{2}\right)^{2}} \ll 1
\end{aligned}
$$

Since, according to Eqs. (8), $\hat{\rho}_{T}$ is proportional to the temperature $\tilde{T}=\int_{0}^{1} \hat{T} \hat{r}^{2} \mathrm{~d} \hat{r}$, it follows from the first Eq. (10) that $\mathrm{d} \hat{\rho}_{T} / \mathrm{d} \tau=O\left(\varepsilon \hat{\rho}_{T}\right)$ has a higher order of smallness than $\overrightarrow{\hat{\rho}}_{T}$ itself. As a consequence, the thermal bow in Eq. (1) can be considered as a parameter, and the system dynamics become essentially decoupled from the thermal part of problem. 
The rotor lateral mode modal coordinates $\vec{w}_{q}=u_{q}+\mathrm{j} v_{q}(q=1,2, \ldots, \infty)$ together with orthogonal modal functions $\eta_{q}(q=1,2, \ldots, \infty)$ can be introduced in a way that the following equations are satisfied:

$$
\begin{aligned}
\vec{r} & =\sum_{k=1}^{\infty} \eta_{q}(l) \vec{w}_{q}(t)+\overrightarrow{\hat{\rho}} \mathrm{e}^{\mathrm{j} \varphi}=\sum_{k=1}^{\infty} \eta_{q}(l)\left(\vec{w}_{q}+\pi_{q} \overrightarrow{\tilde{T}}^{\mathrm{j} \varphi}\right) ; \\
& -\int_{0}^{L} \eta_{q}(l) \frac{M_{1}\left(l, l_{2}\right)}{I\left(l_{2}\right)} \mathrm{d} l=\pi_{q} .
\end{aligned}
$$

In this case, Eq. (1) can be written in modal coordinates as follows:

$$
\begin{aligned}
M_{q} \ddot{\vec{w}}_{q} & +D_{q}\left(\dot{\vec{w}}_{q}+\mathrm{j} \Omega \pi_{q} \overrightarrow{\tilde{T}}^{\mathrm{j} \varphi}\right)+K_{q} \vec{w}_{q} \\
= & M_{q}\left(\vec{e}_{q}+\pi_{q} \overrightarrow{\tilde{T}}\right) \Omega^{2} \mathrm{e}^{\mathrm{j} \varphi}+P_{q} \mathrm{e}^{\mathrm{j} \gamma_{q}} \\
& -\vartheta K_{f}\left(1-\frac{c}{\left|\vec{r}_{2}\right|}\right)(1+\mathrm{j} f) \eta_{q}\left(l_{2}\right) \\
& \times \sum_{n=1}^{\infty} \eta_{n}\left(l_{2}\right)\left(\vec{w}_{n}+\pi_{n} \overrightarrow{\widetilde{T}}^{\mathrm{j} \varphi}\right), \quad q=1,2, \ldots, \infty .
\end{aligned}
$$

Here $M_{q}, D_{q}$ and $K_{q}$ are modal mass, damping and stiffness of the $q$ th mode, $\vec{e}_{q}=\int_{0}^{L} m \vec{e} \eta_{q} \mathrm{~d} l$ is a modal unbalance, $P_{q} \mathrm{e}^{\mathrm{j} \gamma_{q}}=\int_{0}^{L} q \mathrm{e}^{\mathrm{j} \gamma} \eta_{q} \mathrm{~d} l$ is a modal radial side-load force. The analysis of Eqs. (13) which represent the mathematical model of the system, is performed below under the following physical assumptions:

(1) The system of applied radial side-load forces maintains the static position of the rotor very close to the stationary obstacle at the axial location $l_{2}$. The rotor-to-stator contact is intermittent due to the dynamic action of the unbalance.

$$
\begin{gathered}
P_{k} \mathrm{e}^{\mathrm{j} \gamma_{k}}=-\mathrm{j} c \frac{K_{k}}{\kappa_{k}}\left(1-\Delta_{k}\right), \\
P_{q} \mathrm{e}^{\mathrm{j} \gamma_{q}}=\mathrm{j} c \Delta_{q} \frac{K_{q}}{\kappa_{q}} \quad(q \neq k) .
\end{gathered}
$$

In this case, the absolute value of the shaft static displacement at the rub axial location is as follows:

$$
\left|\vec{r}_{2 s t}\right|=c\left(1-\sum_{q=1}^{\infty} \Delta_{q}\right)=c(1-\sigma \Delta),
$$

$\sigma= \begin{cases}1 & \text { for statically "loose" case (no contact), } \\ -1 & \text { for statically "tight" case (contact), }\end{cases}$

where $c \Delta$ is an absolute value of a gap between the rotor static position and the stator. According to physical assumptions, $\Delta \ll 1$. The coefficient $\Delta$ is used below as a measure of smallness.

(2) One mode in particular, the $k$ th mode, for example, governs the contact at the axial location $l_{2}$. It means that this mode delivers a much higher contribution into $\left|\vec{r}\left(l_{2}, t\right)\right|$ than all other modes:

$$
\begin{aligned}
& \eta_{k}\left(l_{2}\right)=\kappa_{k}=O(1), \\
& \eta_{q}\left(l_{2}\right)=\sqrt{\Delta} \kappa_{q}=O(\sqrt{\Delta}) \quad(q \neq k) .
\end{aligned}
$$

In almost all practical situations this is true.

(3) The vertical (imaginary) direction for each mode is chosen opposite to the direction of the corresponding radial side-load force: $\gamma_{q}=$ $3 \pi / 2, q=1,2, \ldots$

New variables are introduced as follows:

$$
\begin{aligned}
\vec{w}_{k}= & \frac{c}{\kappa_{k}}\left[-\mathrm{j}\left(1-\Delta_{k}\right)+\Delta(u+\mathrm{jv})\right], \\
\vec{w}_{q}= & \frac{c}{\kappa_{q}}\left(\mathrm{j} \frac{\Delta_{q}}{\sqrt{\Delta}}+\Delta^{3 / 2} \vec{\Gamma}_{q}\right) \quad(q \neq k), \\
h= & \frac{c-\left|\vec{r}_{2}\right|}{c \Delta} \\
= & \sigma\left[1+\mathrm{v}+\frac{\Delta}{2}\right. \\
& \left.\times\left(2 \sum_{q \neq k} \operatorname{Im} \vec{\Gamma}_{q}-u^{2}+2 s_{T} \mathrm{e}^{\mathrm{j}\left(\varphi+\alpha_{T}\right)}\right)+O\left(\Delta^{2}\right)\right],
\end{aligned}
$$

where $(u+\mathrm{jv})$ and $\Gamma_{q}(q \neq k)$ are dynamic components of Eqs. (13) solutions, $h$ is the 
nondimensional distance between the rotor and an obstacle at the axial location $l_{2}$,

$$
s_{T}=\left|\overrightarrow{\hat{\rho}}\left(l_{2}, t\right)\right| /\left(c \Delta^{2}\right)=O(1), \alpha_{T}=\arg \left(\overrightarrow{\hat{\rho}}\left(l_{2}, t\right)\right) .
$$

With the new set of variables $h, u, \Gamma_{q}(q \neq k)$ using Eqs. (16) and (13) can be rewritten as follows:

$$
\begin{aligned}
& h^{\prime \prime}+n_{k}^{2}\left(1+\vartheta p^{2}\right) h-\sigma n_{k}^{2}=\Delta H+O\left(\Delta^{2}\right), \\
& u^{\prime \prime}+n_{k}^{2} u=\Delta U+O\left(\Delta^{2}\right), \\
& \Gamma_{q}^{\prime \prime}+n_{q}^{2} \Gamma_{q}=-\mathrm{j} \vartheta \sigma n_{q}^{2} p_{q}^{2} h \\
& \quad+\sqrt{\Delta} \kappa_{q}\left[a_{q} \mathrm{e}^{\mathrm{j}\left(\varphi+\alpha_{q}\right)}+\pi_{q *} S_{T} \mathrm{e}^{\mathrm{j}\left(\varphi+\alpha_{T}\right)}\right] \\
& \quad+\Delta W_{q}+O\left(\Delta^{2}\right),
\end{aligned}
$$

where of smallness, but the conditions (19) of the switch are precise.

\section{MECHANICAL RESONANCES AND HEAT GENERATION}

The rotor resonance responses to the unbalance which are considered below are associated with the leading mode, or in mathematical terms, are described by the first two equations (17). It means that the rest of the Eqs. (17) determine only "forced" solutions, which are defined at each sequential approximation by the previous approximation to the solution of the first two equations. Therefore, it is important to consider the latter in

$$
\begin{aligned}
& H=-2 \varsigma_{k} n_{k} h^{\prime} \\
& +\sigma\left[\kappa_{k} a_{k} \sin \left(\varphi+\alpha_{k}\right)+s_{T}\left(\kappa_{k} \pi_{k^{*}}+n_{k}^{2}-1\right) \sin \left(\varphi+\alpha_{T}\right)+\frac{n_{k}^{2} u^{2}}{2}-u^{\prime 2}+\sum_{q \neq k}\left(n_{k}^{2}-n_{q}^{2}\right) \operatorname{Im} \Gamma_{q}\right], \\
& U=-2 \varsigma_{k} n_{k} u^{\prime}+\kappa_{k} a_{k} \cos \left(\varphi+\alpha_{k}\right)+\kappa_{k} \pi_{k^{*} S_{T}} \cos \left(\varphi+\alpha_{T}\right)+\vartheta p_{k}^{2} n_{k}^{2} \sigma h\left(u+\frac{f}{\Delta}\right) \text {, } \\
& W_{q}=-2 \varsigma_{q} n_{q} \Gamma_{q}^{\prime}+\vartheta p_{q}^{2} n_{q}^{2} \sigma h\left(u+\frac{f}{\Delta}\right) \\
& \varsigma_{k, q}=\frac{D_{k, q}}{2 \Delta M_{k, q} \nu_{k, q}}=O(1) \\
& \nu_{k, q}^{2}=\frac{K_{k, q}}{M_{k, q}}, \quad n_{k, q}^{2}=\frac{\nu_{k, q}^{2}}{\Omega^{2}}, \quad a_{k, q} \mathrm{e}^{\mathrm{j} \alpha_{k, q}}=\frac{\vec{e}_{k, q}}{c \Delta^{2}}=O(1), \\
& \frac{f}{\Delta}=O(1), \varphi=\Omega t, " “, ",=\frac{\mathrm{d}}{\mathrm{d} \varphi}, \\
& p_{k, q}^{2}=\frac{K_{f} \kappa_{k, q}^{2}}{K_{k, q}}, p^{2}=\frac{K_{f}}{K_{k}}\left(\kappa_{k}^{2}+\Delta \sum_{q \neq k} \frac{\kappa_{q}^{2} M_{k}}{M_{q}}\right), \\
& \pi_{q} \overrightarrow{\tilde{T}}=c \Delta^{2} \pi_{q *} s_{T} \mathrm{e}^{\mathrm{j} \alpha_{T}}, \pi_{q *}=-\pi_{q} \frac{I\left(l_{2}\right)}{M_{1}\left(l_{2}, l_{2}\right)} .
\end{aligned}
$$

The conditions (3) of the contact/no-contact switch now become very simple:

$$
\vartheta= \begin{cases}0 & \text { if } \sigma h>0 \\ 1 & \text { if } \sigma h \leq 0\end{cases}
$$

Note, that the right-side terms of Eqs. (17) are calculated with an accuracy up to the second order generating approximation, which is defined by neglecting all right-side terms. The second of Eqs. (17) in generating approximation has a simple solution: $u=\rho \cos \theta, \rho^{\prime}=0, \theta^{\prime}=n_{k}$, The first of Eqs. (17) in generating approximation, together with the switch conditions (19), is more complex. Its solution can be built using piecewise integration, and connecting 
conditions at the ends of continuity intervals:

$$
\begin{aligned}
h= & \frac{S}{1+\chi(\hat{\Phi}) p^{2}}\left[\sqrt{1+\chi(\hat{\Phi}) p^{2}\left(1-\frac{1}{S^{2}}\right)} \sin \Xi\right. \\
& \left.+\frac{1}{\sigma S}(1-\cos \Xi)\right]
\end{aligned}
$$

where $S$ is constant referred as an amplitude parameter,

$$
\begin{aligned}
& \Phi=\omega(S) \tau+\alpha \\
& \omega(S)=n_{k}\left[1+\frac{1}{\pi}\left(\left(1+p^{2}\right)^{-1 / 2} \arccos \left(\sigma S \sqrt{1+p^{2}\left(1-\frac{1}{S^{2}}\right)}\right)^{-1}-\arccos \frac{1}{\sigma S}\right)\right]^{-1} \\
& \Xi=\frac{n_{k} \sqrt{1+\chi(\hat{\Phi}) p^{2}}}{\omega}\left\{\Phi-2 \pi\left(\left[\frac{\Phi}{2 \pi}\right]+\chi(\hat{\Phi})\right)\right\} \\
& \hat{\Phi}=\Phi-2 \pi\left[\frac{\Phi}{2 \pi}\right]-2\left(\pi-\arccos \frac{1}{\sigma S}\right) \frac{\omega}{n_{k}} .
\end{aligned}
$$

The relation between $h$ and $S$ (amplitude parameter) and $\Phi$ (phase) is shown graphically in Fig. 3 . It also shows the relation between the amplitude parameter $S$ and overall amplitude $A$. Equations (20) and (21) constitute a variable transformation. This transformation allows to introduce the rotor vertical response amplitude parameter $S$ and "phase" $\Phi$, and is used to convert the original system (17) into the form with three rotating phases $\varphi, \Phi, \theta$ and two slow variables $S, \rho$ :

$$
\begin{aligned}
& S^{\prime}=\Delta Q\left(S, \rho, \Phi, \theta, \varphi, \Gamma_{q}(S, \Phi)\right)+O\left(\Delta^{2}\right) ; \\
& \Phi^{\prime}=\omega-\omega \Delta F\left(S, \rho, \Phi, \theta, \varphi, \Gamma_{q}(S, \Phi)\right)+O\left(\Delta^{2}\right) \\
& \rho^{\prime}=\frac{\Delta U\left(S, \rho, \Phi, \theta, \varphi, \Gamma_{q}(S, \Phi)\right) \sin \theta}{n_{k}}+O\left(\Delta^{2}\right) ; \\
& \theta^{\prime}=n_{k}-\frac{\Delta U\left(S, \rho, \Phi, \theta, \varphi, \Gamma_{q}(S, \Phi)\right) \cos \theta}{\rho n_{k}}+O\left(\Delta^{2}\right) \\
& \varphi^{\prime}=1
\end{aligned}
$$

and a number of noncritical variables $\Gamma_{q}$ :

$$
\begin{gathered}
\Gamma_{q}=-\mathrm{j} \sigma p_{q}^{2} \frac{n_{q}}{\omega} \int_{0}^{\Phi} \chi\left(\hat{\Phi}\left(\Phi_{*}\right)\right) h\left(S, \Phi_{*}\right) \\
\sin \frac{n_{q}\left(\Phi-\Phi_{*}\right)}{\omega} \mathrm{d} \Phi_{*}+O(\Delta)
\end{gathered}
$$

where $Q, F, U, W$ are functions of new variables, determined by the system of Eqs. (17) and $\Phi_{*}$ is an integration variable. Equations (22) have the required format, with three fast rotating phases $\Phi$, $\theta, \varphi$ and two slow variables $S$ and $\rho$. This allows application of the Averaging Method [7]. Note that at this point, the equations are limited to the terms of the first order of smallness, and only small right-sided terms of Eqs. (22) have discontinuities, as they change with the fast rotating phase $\Phi$.

As it results from the expression (21), the ratio $\omega / n_{k}$ is contained within the following limits:

$$
\begin{aligned}
& 1 \leq \frac{\omega}{n_{k}} \leq \frac{2}{1+1 / \sqrt{1+p^{2}}} \text { if } \sigma=+1, \\
& \frac{2}{1+1 / \sqrt{1+p^{2}}} \leq \frac{\omega}{n_{k}} \leq \sqrt{1+p^{2}} \text { if } \sigma=-1 .
\end{aligned}
$$

These inequalities, together with Fourier analysis of the right-side terms of Eqs. (22), show that possible resonances occur when:

$$
\begin{aligned}
& 1-n_{k}=0, \quad 1-i \omega=0(i=1,2,3, \ldots), \\
& 2 n_{k}-\omega=0 \quad(\text { for } \sigma=-1) .
\end{aligned}
$$

The analysis of Eqs. (22) from the standpoint of balance between the supplied and dissipated energies, allows the stationary resonance solutions for the case of $1-n_{k}=0,1-i \omega=0(i=1,2,3, \ldots)$, or for the case of a combinational resonance: $1-n_{k}=0$ and $2 n_{k}-\omega=0$. The first resonance occurs when the rotative speed of the shaft $\Omega$ is close to the rotor 


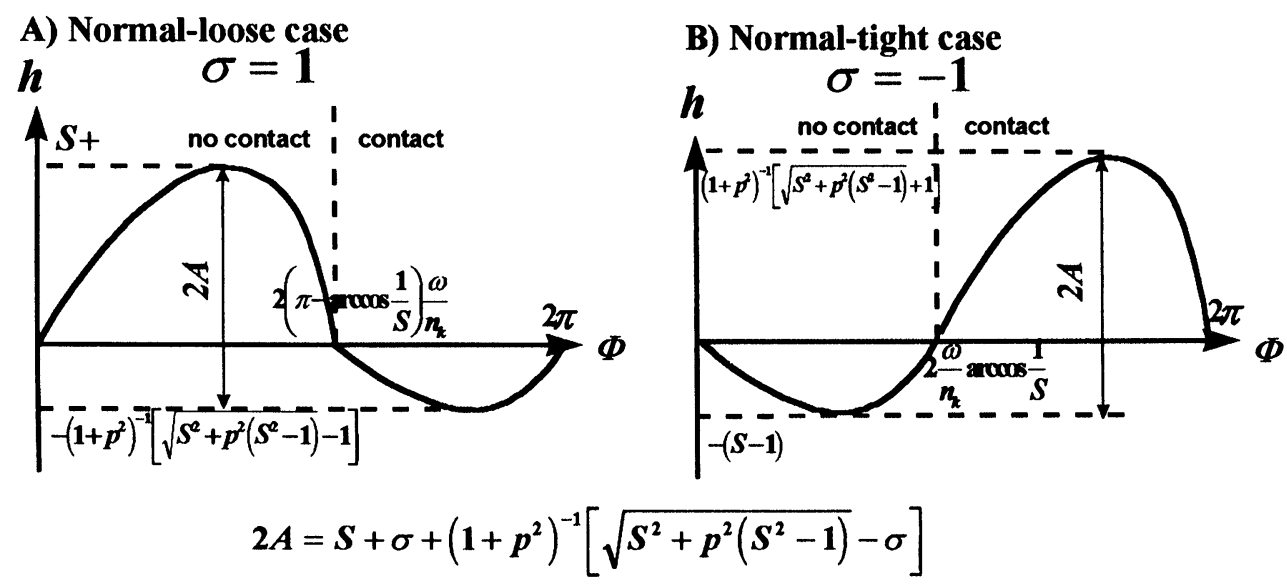

FIGURE 3 Variable nondimensional distance $h=\left(c-\left|\vec{r}_{2}\right|\right) / c \Delta$ as a function of phase $\Phi$.

leading mode natural frequency $v_{k}$. It could either be accompanied by the vertical resonance $2 n_{k}-\omega=0$ or not. This regime, referred to as a horizontal mode resonance, is described in detail in Taylor (1924). Important thing to know about horizontal mode resonance is that, due to the symmetric heating, the thermal bow does not occur. The sequence of resonances $1-i \omega\left(S_{a}\right)=0 \quad(i=$ $1,2,3, \ldots)$, referred to as vertical mode resonances, is of interest because it creates uneven heating which results in a thermal bow. The resonance frequency equation according to Eqs. (21) determines the zeroth approximation $S_{a}$ to the slow variable $S$ as a function of the ratio $\Omega / i \nu_{k}$ in the corresponding resonance zone. A simple analysis of Eqs. (21), together with inequalities (24), shows that for each value of $i=1,2, \ldots$ it can be satisfied only within the range of rotative speeds determined by the following inequality:

$$
\begin{aligned}
& i \nu_{k} \leq \Omega \leq \frac{2 i \nu_{k}}{1+1 / \sqrt{1+p^{2}}} \text { if } \sigma=+1 \\
& \frac{2 i \nu_{k}}{1+1 / \sqrt{1+p^{2}}} \leq \Omega \leq i \nu_{k} \sqrt{1+p^{2}} \text { if } \sigma=-1 .
\end{aligned}
$$

This means that the main, $1 \times$ (synchronous) regime of rotor vibrations $(i=1)$ occurs at rotative speeds higher then unaltered resonance frequency $\nu_{k}$ of the leading $k$ th mode, the subsynchronous $1 / 2 \times$ regime $(i=2)$ occurs at rotative speeds higher then $2 \nu_{k}$, and so on. In the case of normal-loose situation, $(\sigma=+1)$ the maximum rotative frequency of the corresponding resonance regime is lower then $2 i \nu_{k}$, while in the normal-tight situation $2 i \nu_{k}$ is a minimal rotative frequency. This agrees with practical observations of rubbing rotor behavior (Choi and Noah, 1987). The parameter $p$, which affects the width of the frequency band for each regime, characterizes the stiffening effect of the rotor-to-stator contact. Equations (22), after averaging in proximities of vertical resonances (see details in Zhuravlev (1978)), allow for the following stable stationary solutions:

$$
\begin{aligned}
& i \Phi=\varphi+\gamma, \\
& \gamma=\Theta+\vartheta \\
& +\operatorname{sign}(G) \arccos \frac{\pi k\left(1-n_{k}^{2}\right)\left[\left(1+p^{2}\right) n_{k}^{2}-1\right] \varsigma_{k} G}{b p^{2}\left(\sqrt{1-1 / S_{a}^{2}} \cos \vartheta+n_{k} \sigma / S_{a} \sin \vartheta\right)} \\
& \quad i=1,2,3, \ldots \\
& \vartheta=\frac{1}{n_{k}}\left(\pi-\arccos \frac{1}{S_{a}}\right), \\
& G\left(S_{a}, p^{2}\right) \\
& =\left\{S_{a}+\frac{p^{2} \sigma}{\pi\left(1+p^{2}\right) i n_{k}}\left[\sqrt{1-\frac{1}{S_{a}^{2}}}-\frac{\sigma n_{k}}{S_{a}}(i \pi-\vartheta)\right]\right\}
\end{aligned}
$$


where $S_{a}$ is a function of the rotative speed, determined by the solution of resonance equation $1-i \omega\left(S_{a}\right)=0(i=1,2,3, \ldots), \quad \vec{b}=b \mathrm{e}^{\mathrm{j} \Theta}=\kappa_{k} a_{k}$ $\mathrm{e}^{\mathrm{j} \alpha_{k}}+S_{T}\left(\kappa_{k} \pi_{k^{*}}+n_{k}^{2}-1\right) \mathrm{e}^{\mathrm{j} \alpha_{T}}$ is an equivalent vector of unbalance which includes a component due to the thermal bow. It defines the series of $1 \times, 1 / 2 \times, 1 / 3 \times, \ldots$ regimes in the rotative speed bands roughly described by the inequality (26). The relationship of the vertical response phase $\Phi-(\varphi+\vartheta / i)$ and overall amplitude $A$ with rotative speed ratio for the vertical modes, calculated for a particular set of parameters $\sigma, p, \varsigma_{k}$ and $b$, is presented in Fig. 4. Based on the resonance solutions (27) and expressions (11), the heat generating vector $\langle\overrightarrow{\hat{g}}\rangle$ can be calculated:

$$
\begin{aligned}
\langle\overrightarrow{\hat{g}}\rangle= & \mathrm{j} f \Omega c \Delta K_{f} \sigma \frac{\sqrt{S_{a}^{2}+p^{2}\left(S_{a}^{2}-1\right)}}{2 \pi i\left(1+p^{2}\right)} \\
& \times\left\{\mathrm{j} \cos \delta\left(1-\mathrm{e}^{-2 \mathrm{j} \vartheta}\right)+\frac{1+\mathrm{j} \cos \delta}{n_{k}^{2}\left(1+p^{2}\right)}\right. \\
& \left.\times\left[1-\mathrm{e}^{-2 \mathrm{j} \vartheta}\left(\cos 2 \delta-\mathrm{j} n_{k} \sqrt{1+p^{2}} \sin 2 \delta\right)\right]\right\} \mathrm{e}^{\mathrm{j} \gamma}, \\
\delta= & \arccos \left(1 /\left(\sigma S_{a} \sqrt{1+p^{2}\left(1-1 / S_{a}^{2}\right)}\right)\right),
\end{aligned}
$$

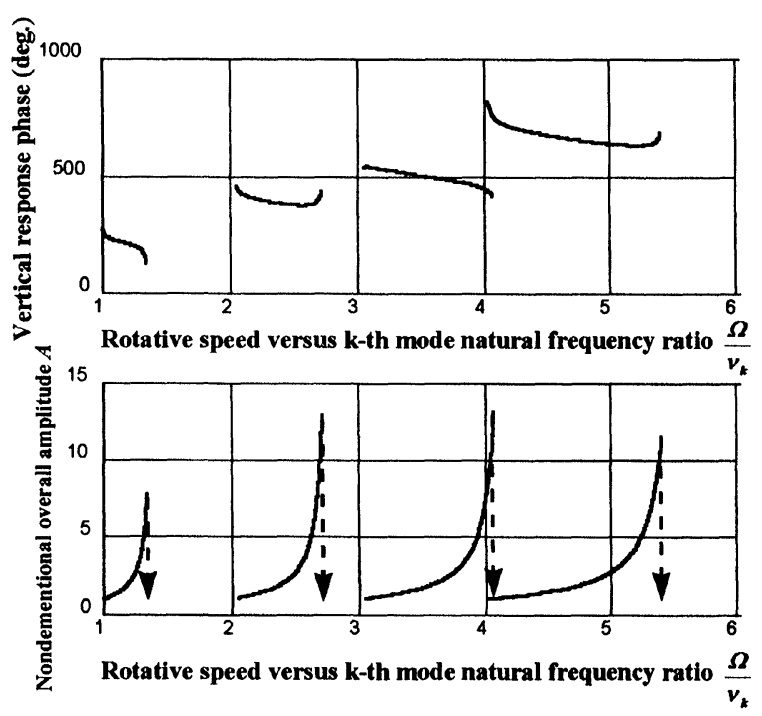

FIGURE 4 Phase and nondimensional amplitude of the rotor-to-stator distance versus rotative speed to natural frequency ratio for the series of $1 \times, \frac{1}{2} \times, \frac{1}{3} \times, \frac{1}{4} \times$ resonances. Calculations are made for the parameters $\sigma=1, p=2, b / \varsigma_{k}=4$. where $S_{a}$ is determined by the second of Eqs. (25) and Eqs. (21) as a function of rotative speed $\Omega$. Since $\langle\overrightarrow{\hat{g}}\rangle$ is essentially the forcing function in Eq. (10) for the thermal bow, its behavior determines the behavior of the thermal bow. Figure 5 depicts $\langle\overrightarrow{\hat{g}}\rangle$ in a polar plot format for different resonance regimes $i=1,2,3,4$.

\section{SUMMARY AND CLOSING REMARKS}

This paper outlines the modeling of thermal/ mechanical effects of one of the most destructive malfunctions in rotating machinery: the rotorto-stator rub. The thermal/mechanical problem is partially uncoupled by the assumption that the thermal process is relatively slow. As a result, the rotor thermal bow remains in the mechanical equations as a parameter which can be considered a constant. The combination of the Averaging Method and the assumption that the thermal processes are quasi-static allows the heat transfer problem to reduce to a vectorial ordinary differential equation (Eq. (11)), with the heat generating equivalent vector as a forcing function. This equation allows a realistic estimate of the thermal

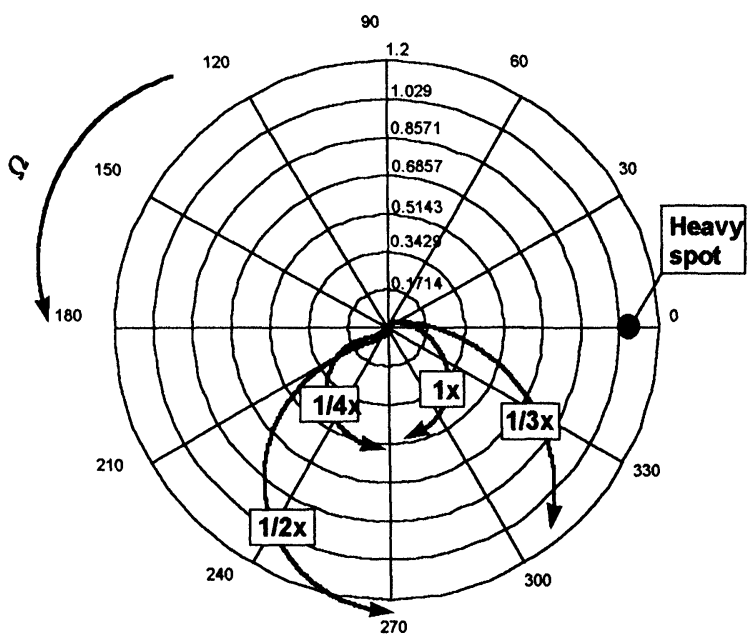

FIGURE 5 Heat generating vector $\langle\overrightarrow{\hat{g}}\rangle$ versus rotative speed to the natural frequency ratio in the polar plot format. Heavy spot indicates the position of equivalent unbalance vector $\vec{b}, \sigma=1, p=2, b / \varsigma_{k}=4$. 
bending mode (Fig. 1 and Eqs. (9)), and can be applied not only to rub-related heating. The other possible application is an effect of journal bearing differential heating, described in the paper by Keogh and Morton (1993).

The heat generating equivalent vector for the rub is calculated by application of the discontinues variable transformation and resonance version of the Averaging Method to the mechanical part of the problem. Based on the heat generating equivalent vector behavior, predictions can be made on the one of three possible thermal bow behaviors:

- Asymptotic approach to the equilibrium state of the thermal bow.

- Increasing spiraling motion of the thermal bow in the direction opposite to rotation.

- Slow oscillations of the thermal bow.

The analytical algorithm described in this paper (Eqs. (20), (21), (23) and (27)) has a high potential as a valuable research and prediction tool for investigating rub and thermal effects in rotating machinery.

\section{References}

Bently, D.E. (1974), Forced subrotative speed dynamic action of rotating machinery, ASME Paper, 74-DET-16, Petroleum Mechanical Engineering Conference, Dallas, Texas.

Black, H.F. (1968), Interaction on a whirling rotor with a vibrating stator across a clearance annulus, J. Mech. Eng. Sci., 10(1), 1-12.

Bogolubov, N.N. and Mitropolsky, U.A. (1974), Asymptotic methods in the theory of nonlinear oscillations (in Russian), Nauka, Moskva.

Childs, D.W. (1979), Rub-induced parametric excitation in rotors, ASME Journal of Mechanical Design, ASME Paper 79-WA, DE-14. ASME Trans., Vol. 101, pp. 649-654.

Childs, D.W. (1982), Fractional frequency rotor motion due to nonsymmetric clearance effects, Journal of Engineering for Power, 533-541.

Choi, Y.S. and Noah, S.T. (1987), Nonlinear steady-state response of a rotor-support system, Journal of Vibration, Acoustics, Stress and Reliability in Design, 255-261.

Dimarogonas, A.D. (1973), Newkirk effect: Thermally induced dynamic instability of high speed rotors, ASME Paper 73-GT26, Gas Turbine Conference, Washington, DC.

Dimarogonas, A.D. (1974), A study of the Newkirk effect in turbomachinery, WEAR, 28, 1974, 369-382.

Dimarogonas, A.D. and Paipetis, S.A. (1983), Analytical Methods in Rotor Dynamics, Applied Science Publishers, London and New York.
Goldman, P. and Muszynska, A. (1994a), Dynamic effects in mechanical structures with gaps and impacting: order and chaos, Transactions of ASME, Journal of Vibration and Acoustics, 116, 541-547.

Goldman, P. and Muszynska, A. (1994b), Resonances in the system of the interacted sources of vibration. Formulation of problem and general results, International Journal for Nonlinear Mechanics, 29(1), 49-63.

Goldman, P. and Muszynska, A. (1995a), Rotor-to-stator-rubrelated, thermal/mechanical effects in rotating machinery, Chaos, Solitons, and Fractals, Vol. 5, No. 9.

Goldman, P. and Muszynska, A. (1995b), Smoothing technique for rub or looseness-related rotor dynamic problems, Proceedings of 1995 Design Engineering Conferences, Vibration of Nonlinear Random and Time-Varying Systems, DEVol. 84-1.

Goodier, J.N. (1958), Formulas for overall thermoelastic deformation, Proc. 3rd Int. Congr. Appl. Mech., John Wiley, New York, p. 343.

Hashemi, Y. (1984), Vibration problems with thermally induced distortions in turbine-generators rotors, vibrations in rotating machinery, Third Int. Conf. Proceedings, IMechE, C271/84, York, U.K.

Kellenberger, W. (1979), Spiral vibrations due to the seal rings in turbogenerators. Thermally induced interaction between rotor and stator, ASME Paper 79-DET-61, Design Eng. Tech. Conf., St. Louis.

Keogh, P.S. and Morton, P.G. (1993), Journal bearing differential heating evaluation with influence on rotor dynamic behavior, Proceedings of the Royal Society, London, A441.

Kroon, R.P. and Williams, W.A. (1939), Spiral vibration of rotating machinery, Proc. 5th Int. Congr. Appl. Mech., John Wiley, New York, p. 712.

Muszynska, A. (1989), Rotor-to-stationary element rub-related vibration phenomena in rotating machinery, literature survey, The Shock and Vibration Digest, 21(3), 3-11.

Muszynska, A., Franklin, W.D. and Hayashida, R.D. (1990), Rotor-to-stator partial rubbing and its effects on rotor dynamic response, The Sixth Workshop on Rotordynamic Instability Problem in High Performance Turbomachinery, NASA CP 3122, College Station, Texas, pp. 345-362.

Muszynska, A. (1993), Thermal/mechanical effect of rotor-tostator rubs in rotating machinery, Vibration of Rotating Systems, DE-vol. 60, ASME Design Technical Conf., Albuquerque, New Mexico.

Natho, N.S. and Crenwelge, O.E. (1983), Case history of a steam turbine rotordynamic problem: Theoretical versus experimental results, Vibration Institute Proceedings, Machinery Vibration Monitoring \& Analysis, pp. 81-89.

Newkirk, B.L. (1926), Shaft rubbing, Mech. Eng., 48, 830.

Petchenev, A. and Fiddling, A. (1992), Hierarchy of the resonant motions of vibroimpacting system excited by the inertia source with limited power (in Russian), Mechanika Tverdogo Tela, No. 4.

Smalley, A.J. (1987), The Dynamic response of rotors to rubs during startup, Rotating Machinery Dynamics, Vol. 2, Eds. A. Muszynska and J.C. Simonis.

Sweets, W.J. (1966), Analysis of rotor rubbing, GE Technical Information Series, DF-66-LS-70.

Taylor, H.D. (1924), Rubbing shafts above and below resonant speed, GE Technical Information Series. No. 16709.

Zhuravlev, B.F. (1978), The equations of motion for the systems with ideal one sided restrictions (in Russian), PMM (Applied Mathematics and Mechanics), Vol. 42, No. 5, pp. 781-788. 


\section{ait \\ ENERGY MATERIALS}

M A N E Y publishing

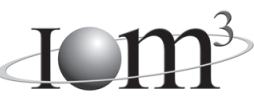

\section{Materials Science \& Engineering for Energy Systems}

Maney Publishing on behalf of the Institute of Materials, Minerals and Mining

The Institute of Materials, Minerals \& Mining

Economic and environmental factors are creating ever greater pressures for the efficient generation, transmission and use of energy. Materials developments are crucial to progress in all these areas: to innovation in design; to extending lifetime and maintenance intervals; and to successful operation in more demanding environments. Drawing together the broad community with interests in these areas, Energy Materials addresses materials needs in future energy generation, transmission, utilisation, conservation and storage. The journal covers thermal generation and gas turbines; renewable power (wind, wave, tidal, hydro, solar and geothermal); fuel cells (low and high temperature); materials issues relevant to biomass and biotechnology; nuclear power generation (fission and fusion); hydrogen generation and storage in the context of the 'hydrogen economy'; and the transmission and storage of the energy produced.

As well as publishing high-quality peer-reviewed research, Energy Materials promotes discussion of issues common to all sectors, through commissioned reviews and commentaries. The journal includes coverage of energy economics and policy, and broader social issues, since the political and legislative context influence research and investment decisions.

\section{CALL FOR PAPERS}

Contributions to the journal should be submitted online at http://ema.edmgr.com

To view the Notes for Contributors please visit: www.maney.co.uk/journals/notes/ema

Upon publication in 2006, this journal will be available via the Ingenta Connect journals service. To view free sample content online visit: www.ingentaconnect.com/content/maney

For further information please contact:

Maney Publishing UK

Tel: +44 (0)113 2497481 Fax: +44 (0)1132486983 Email: subscriptions@maney.co.uk

or

Maney Publishing North America

Tel (toll free): 8662975154 Fax: 6173546875 Email: maney@maneyusa.com

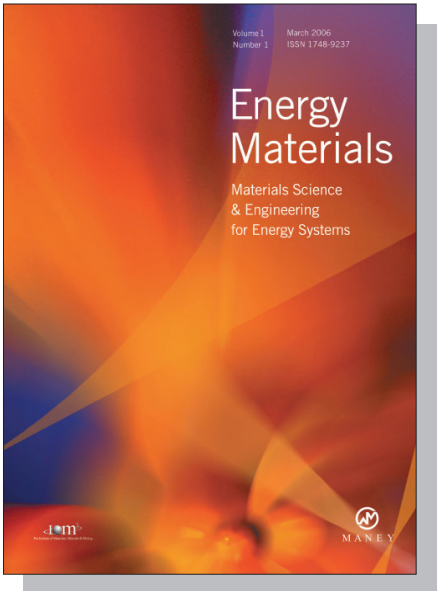

EDITORS

Dr Fujio Abe

NIMS, Japan

Dr John Hald, IPL-MPT, Technical University of Denmark, Denmark

Dr R Viswanathan, EPRI, USA

\section{SUBSCRIPTION INFORMATION}

Volume 1 (2006), 4 issues per year

Print ISSN: 1748-9237 Online ISSN: 1748-9245

Individual rate: $£ 76.00 / U S \$ 141.00$

Institutional rate: $£ 235.00 /$ US $\$ 435.00$

Online-only institutional rate: $£ 199.00 / U S \$ 367.00$

For special $\mathrm{IOM}^{3}$ member rates please email

subscriptions@maney.co.uk

\section{For further information or to subscribe online please visit www.maney.co.uk}



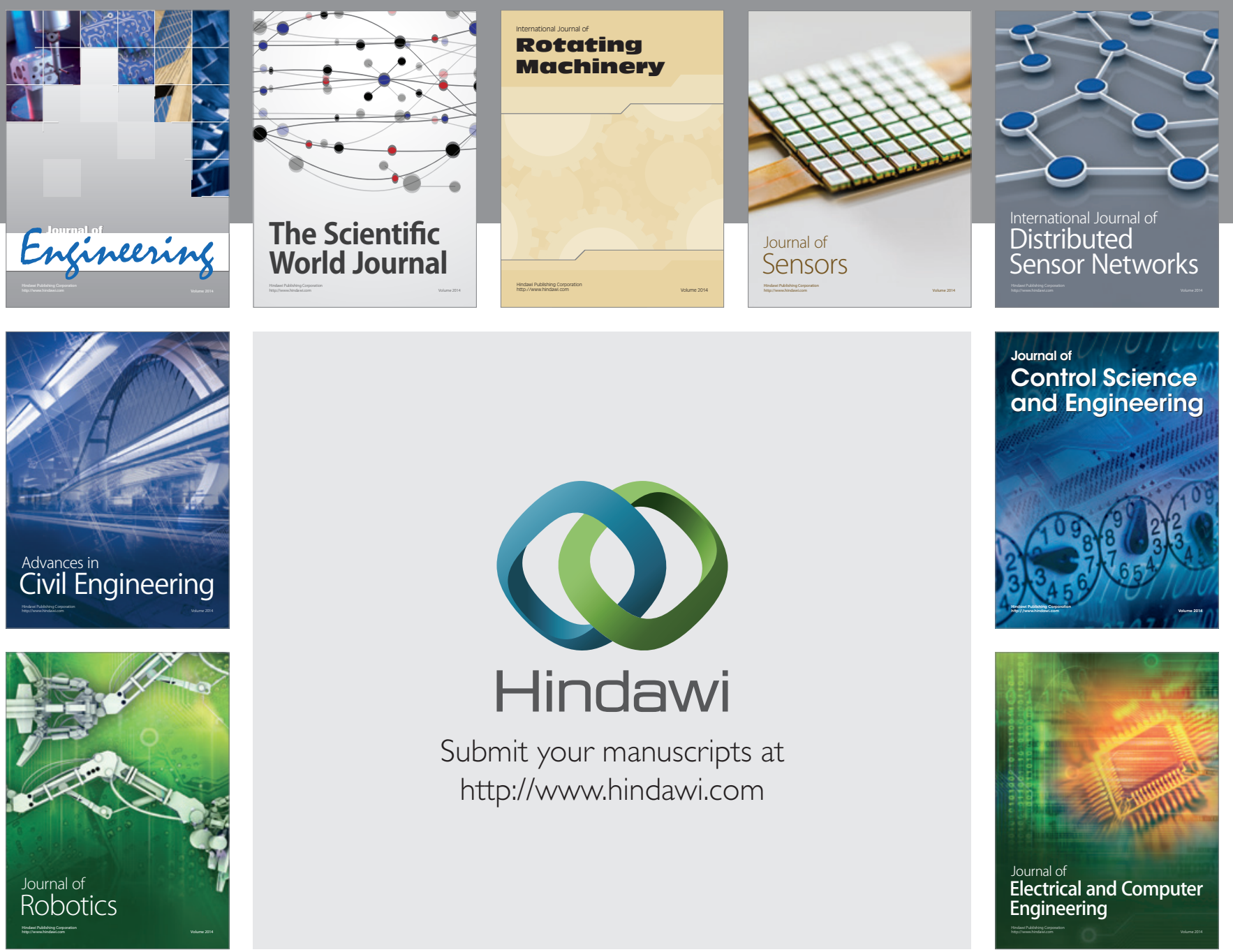

Submit your manuscripts at

http://www.hindawi.com
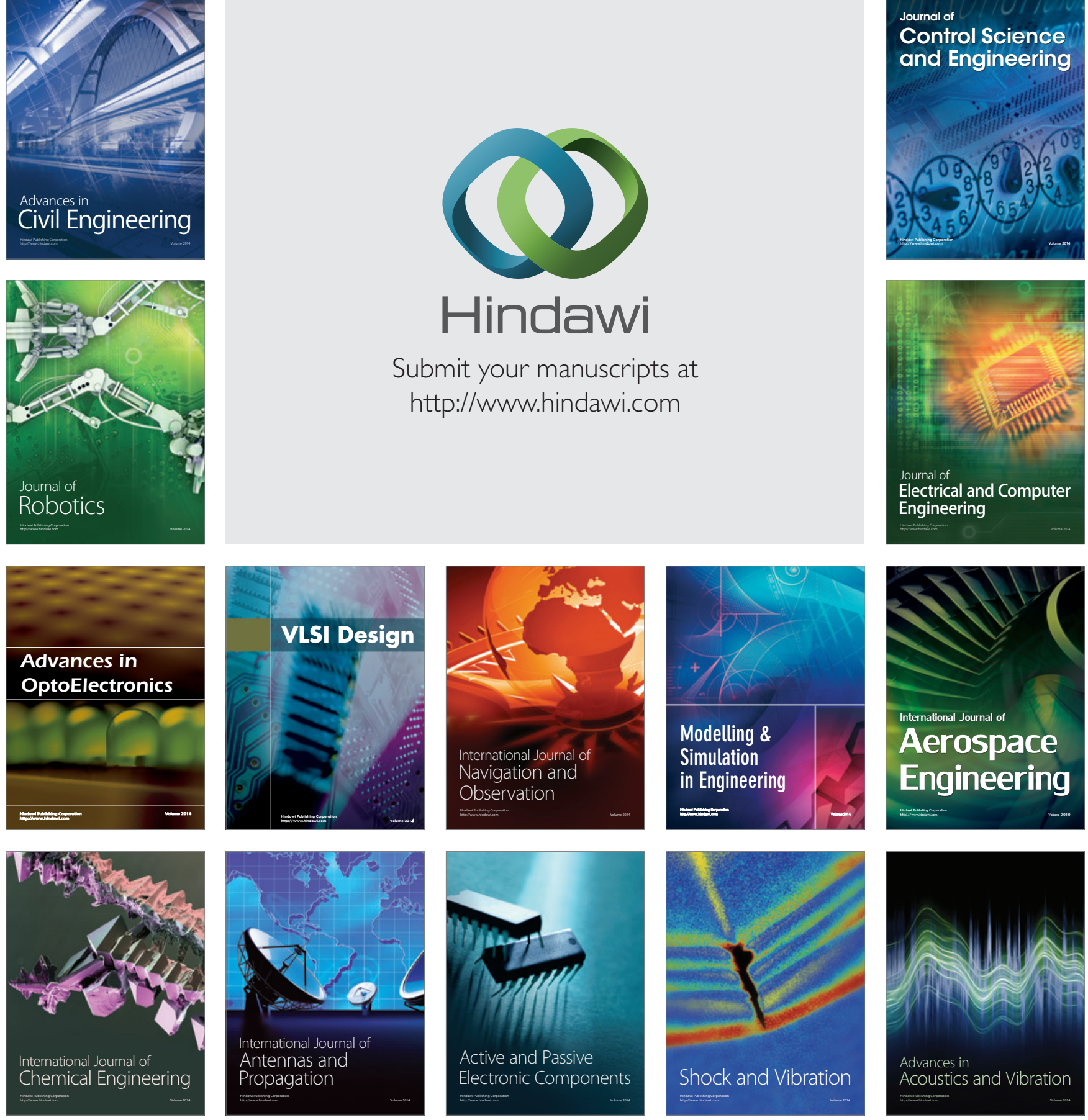